\title{
Symmetry Classification and Exact Solutions of a Variable Coefficient Space-Time Fractional Potential Burgers' Equation
}

\author{
Manoj Gaur and K. Singh \\ Department of Mathematics, Jaypee University of Information Technology, Waknaghat, Solan 173234, India \\ Correspondence should be addressed to K. Singh; karan_jeet@yahoo.com
}

Received 29 April 2016; Revised 13 July 2016; Accepted 9 August 2016

Academic Editor: Wen-Xiu Ma

Copyright ( $\odot 2016$ M. Gaur and K. Singh. This is an open access article distributed under the Creative Commons Attribution License, which permits unrestricted use, distribution, and reproduction in any medium, provided the original work is properly cited.

We investigate the symmetry properties of a variable coefficient space-time fractional potential Burgers' equation. Fractional Lie symmetries and corresponding infinitesimal generators are obtained. With the help of the infinitesimal generators, some group invariant solutions are deduced. Further, some exact solutions of fractional potential Burgers' equation are generated by the invariant subspace method.

\section{Introduction}

Partial differential equations (PDEs) have a wide range of applications in many fields, such as physics, engineering, and chemistry, which are fundamental for the mathematical formulation of continuum models $[1,2]$. Burgers' equation is a one-dimensional nonlinear partial differential equation, which is a simple form of the one-dimensional NavierStokes equation. It was presented for the first time in a paper in 1940s by Burgers. Later, Burgers' equation was studied by Cole [3] who gave a theoretical solution, based on Fourier series analysis, using the appropriate initial and boundary conditions. Burgers' equation has a large variety of applications in the modelling of water in unsaturated soil, dynamics of soil water, statistics of flow problems, mixing and turbulent diffusion, cosmology, and seismology $[3,4]$.

Recently, fractional differential equations have found extensive applications in many fields. Many important phenomena in viscoelasticity, electromagnetics, material science, acoustics, and electrochemistry are elegantly described with the help of fractional-order differential equations [4-9]. It has been revealed that the nonconservative forces can be described by fractional differential equations. Therefore, as most of the processes in the real physical world are nonconservative, the fractional calculus can be used to describe them. Fractional integrals and derivatives also appear in the theory of control of dynamical systems, when the controlled system and/or the controller is described by a fractional differential equation $[10,11]$. In the last few decades, the subject of the fractional calculus has caught the consideration of many researchers who contributed to its development. Recently, some analytical and numerical methods have been introduced to solve a fractional-order differential equation $[7,8,11-24]$. However, all the methods have insufficient development as they allow one to find solutions only in case of linear equations and for some isolated examples of nonlinear equations $[4,7,8,11,23,24]$.

It is very well known that the Lie group method is the most effective technique in the field of applied mathematics to find exact solutions of ordinary and partial differential equations $[25,26]$. However, this approach is not yet applied much to investigate symmetry properties of fractional differential equations (FDEs). To the best of our knowledge, there are a few papers (e.g., $[17,26-32])$ in which Lie symmetries and similarity solutions of some fractional differential equations have been discussed by some researchers. More recently, Jumarie [33] proposed the modified RiemannLiouville derivative and Jumarie-Lagrange method [34], after which a generalized fractional characteristic method and a fractional Lie group method have been introduced by $\mathrm{Wu}$ 
$[32,35]$ in order to solve a fractional-order partial differential equation.

In this paper, we intend to apply Lie group method to solve the space-time fractional potential Burgers' equation of the form

$$
\begin{aligned}
& { }_{0} D_{t}^{\alpha} u=f(t){ }_{0} D_{x}^{2 \beta} u+g(t)\left({ }_{0} D_{x}^{\beta} u\right)^{2}, \\
& \quad x \in(0, \infty), t>0,0<\alpha, \beta<1,
\end{aligned}
$$

where ${ }_{0} D_{t}^{\alpha} u$ and ${ }_{0} D_{x}^{\beta} u$ are the modified Riemann-Liouville derivatives with respect to time and space variables, respectively, and $f(t)$ and $g(t)$ are arbitrary functions of $t$. Equation (1) is connected to the fractional Burgers' equation by the well-known Hopf-Cole transformations and is a generalization of the time-fractional Burgers' equation with constant coefficients examined by $\mathrm{Wu}$ [32]. This work is based on some basic elements of fractional calculus, with special emphasis on the modified Riemann-Liouville type derivative. The paper is organized as follows. In Section 2, we briefly describe some definitions and properties of fractional calculus. In Section 3, we obtain the symmetries for Burgers' equation having six-dimensional Lie algebra. In Section 4, we analyze the reduced systems and find some invariant solutions of (1). Section 5 contains application of invariant subspace method on fractional Burgers' equation (1). Finally, a conclusion is given in Section 6.

\section{Some Concepts from Fractional Calculus}

In this paper, the modified Riemann-Liouville derivative proposed by Jumarie [33] has been adopted. Some definitions are given which have been used in this work.

2.1. Fractional Riemann-Liouville Integral. The fractional Riemann-Liouville integral of a continuous (but not necessarily differentiable) real valued function $f(x)$ with respect to $(d x)^{\alpha}$ is defined as $[7,33,36]$

$$
\begin{aligned}
{ }_{0} I_{x}^{\alpha} f(x) & =\frac{1}{\Gamma(\alpha)} \int_{0}^{x}(x-t)^{\alpha-1} f(t) d t \\
& =\frac{1}{\Gamma(1+\alpha)} \int_{0}^{x} f(t)(d t)^{\alpha}, \quad 0<\alpha \leq 1 .
\end{aligned}
$$

The fractional integral with respect to $(d t)^{\alpha}$ was introduced by Jumarie [36] in order to study the fractional derivative of nondifferentiable functions in modified Riemann-Liouville sense. Here we are fully in Leibniz framework; that is to say $(d x)^{\alpha}$ denote finite increment in fractional sense. As a result, we shall be able to duplicate, in a straightforward manner, most of the known standard formulae by merely making the substitution $(d x)^{\alpha} \rightarrow d x$.

2.2. Riemann-Liouville Fractional Derivative. The fractional Riemann-Liouville derivative of $f(x)$ is defined as [7]

$$
{ }_{0} D_{x}^{\alpha} f(x)= \begin{cases}\frac{d^{n} f}{d x^{n}}, & \alpha=n \in \mathbb{N} \\ \frac{1}{\Gamma(n-\alpha)} \frac{d^{n}}{d x^{n}} \int_{0}^{x}(x-t)^{n-\alpha-1} f(t) d t, & n-1<\alpha<n, n \in \mathbb{N} .\end{cases}
$$

2.3. Modified Riemann-Liouville Derivative. Through the fractional Riemann-Liouville integral, Jumarie [33] proposed the modified Riemann-Liouville derivative of $f(x)$ as

$$
\begin{array}{r}
{ }_{0} D_{x}^{\alpha} f(x) \\
=\frac{1}{\Gamma(n-\alpha)} \frac{d^{n}}{d x^{n}} \int_{0}^{x}(x-t)^{n-\alpha-1}(f(t)-f(0)) d t, \\
n-1<\alpha<n, n \in \mathbb{N} .
\end{array}
$$

2.4. Some Useful Formulae. Here, some properties of modified Riemann-Liouville derivative are given which have been used in this paper:

(i) $d f(x)={ }_{0} D_{x}^{\alpha} f(x)(d x)^{\alpha} / \Gamma(1+\alpha), \alpha>0$.

(ii) ${ }_{0} D_{t}^{\alpha}(u(t) v(t))=\left({ }_{0} D_{t}^{\alpha} u(t)\right) v(t)+u(t)\left({ }_{0} D_{t}^{\alpha} v(t)\right), 0<$ $\alpha<1$.

(iii) ${ }_{0} D_{t}^{\alpha} f(x(t))=(d f / d x)\left({ }_{0} D_{t}^{\alpha} x(t)\right), 0<\alpha<1$, given that $d f / d x$ exists.

(iv) ${ }_{0} D_{x}^{\alpha} x^{\beta}=(\Gamma(1+\beta) / \Gamma(1+\beta-\alpha)) x^{\beta-\alpha}, 0<\alpha<1$, $x>0$, and $\beta>-1$.
(v) $\int(d x)^{\beta}=x^{\beta}, 0<\beta \leq 1$.
(vi) $\Gamma(1+\beta) d x=d^{\beta} x$.

The above formulae and details thereof along with the scope of applications and limitations can be found in [33,37].

2.5. Characteristic Method for Fractional-Order Differential Equations. Applying the fractional chain rule proposed by Jumarie [33], we have

$$
\begin{aligned}
d u= & \frac{1}{\Gamma(1+\beta)} \frac{\partial^{\beta} u(x, t)}{\partial x^{\beta}}(d x)^{\beta} \\
& +\frac{1}{\Gamma(1+\alpha)} \frac{\partial^{\alpha} u(x, t)}{\partial t^{\alpha}}(d t)^{\alpha}, \quad 0<\alpha, \beta \leq 1 .
\end{aligned}
$$

$\mathrm{Wu}[32,35]$ extended the characteristic method of firstorder linear partial differential equation to a linear fractional differential equation of the form

$$
\begin{aligned}
a(x, t) \frac{\partial^{\beta} u(x, t)}{\partial x^{\beta}}+b(x, t) \frac{\partial^{\alpha} u(x, t)}{\partial t^{\alpha}}=c(x, t) & \\
& 0<\alpha, \beta \leq 1
\end{aligned}
$$


and introduced the fractional characteristic equations [32] as given herein:

$$
\frac{(d x)^{\beta}}{\Gamma(1+\beta) a(x, t)}=\frac{(d t)^{\alpha}}{\Gamma(1+\alpha) b(x, t)}=\frac{d u}{c(x, t)} .
$$

On solving the characteristic equations (7) for various infinitesimal generators of (1), one can obtain the similarity variables and reduction of (1) to an ODE.

\section{Symmetry Classification of (1)}

Herein, we investigate the symmetries and reductions of space-time fractional potential Burgers' equation (1). A fractional Lie symmetry of (1) is a continuous group of point transformations of independent and dependent variables which leaves (1) invariant. form

Let us assume that (1) admits the Lie symmetries of the

$$
\begin{aligned}
\frac{\widetilde{x^{\beta}}}{\Gamma(1+\beta)} & =\frac{x^{\beta}}{\Gamma(1+\beta)}+\epsilon \xi(x, t, u)+o\left(\epsilon^{2}\right), \\
\frac{\widetilde{t^{\alpha}}}{\Gamma(1+\alpha)} & =\frac{t^{\alpha}}{\Gamma(1+\alpha)}+\epsilon \tau(x, t, u)+o\left(\epsilon^{2}\right), \\
\widetilde{u} & =u+\epsilon \eta(x, t, u)+o\left(\epsilon^{2}\right),
\end{aligned}
$$

where $\epsilon$ is the group parameter and $\xi, \tau$, and $\eta$ are the infinitesimals of the transformations for the independent and dependent variables, respectively. A group invariant solution of space-time fractional potential Burgers' equation (1) is a solution which can be mapped into another solution of (1) under the point transformations (8). The associated Lie algebra of infinitesimal symmetries of (1) is then the fractional vector field of the form

$$
V=\xi(x, t, u) \frac{\partial^{\beta}}{\partial x^{\beta}}+\tau(x, t, u) \frac{\partial^{\alpha}}{\partial t^{\alpha}}+\eta(x, t, u) \frac{\partial}{\partial u} .
$$

The fractional second-order prolongation of (9) is given by

$$
\begin{aligned}
p{ }^{(2)} V= & \xi(x, t, u) \frac{\partial^{\beta}}{\partial x^{\beta}}+\tau(x, t, u) \frac{\partial^{\alpha}}{\partial t^{\alpha}} \\
& +\eta(x, t, u) \frac{\partial}{\partial u}+\eta^{t} \frac{\partial}{\partial\left({ }_{0} D_{t}^{\alpha} u\right)} \\
& +\eta^{x} \frac{\partial}{\partial\left({ }_{0} D_{x}^{\beta} u\right)}+\eta^{t t} \frac{\partial}{\partial\left({ }_{0} D_{t}^{2 \alpha} u\right)} \\
& +\eta^{x t} \frac{\partial}{\partial\left({ }_{0} D_{t}^{\alpha}\left({ }_{0} D_{x}^{\beta} u\right)\right)}+\eta^{x x} \frac{\partial}{\partial\left({ }_{0} D_{x}^{2 \beta} u\right)}
\end{aligned}
$$

Now, for the invariance of (1) according to [26] under (8), we must have

$$
\left.\operatorname{pr}^{(2)} V([\Delta u])\right|_{[\Delta u]=0}=0
$$

where $[\Delta u]={ }_{0} D_{t}^{\alpha} u-f(t){ }_{0} D_{x}^{2 \beta} u-g(t)\left({ }_{0} D_{x}^{\beta} u\right)^{2}$ or equivalently where

$$
\begin{aligned}
& \left(\eta^{t}-f(t) \eta^{x x}-2 g(t)\left({ }_{0} D_{x}^{\beta} u\right) \eta^{x}\right. \\
& \quad-\tau\left({ }_{0} D_{t}^{\alpha} f(t)\right){ }_{0} D_{x}^{2 \beta} u \\
& \left.\quad-\tau\left({ }_{0} D_{t}^{\alpha} g(t)\right)\left({ }_{0} D_{x}^{\beta} u\right)^{2}\right)\left.\right|_{[\Delta u]=0}=0 .
\end{aligned}
$$

The generalized fractional prolongation vector fields $\eta^{x}, \eta^{t}$, and $\eta^{x x}$ are given by

$$
\begin{aligned}
\eta^{x} & ={ }_{0} D_{x}^{\beta} \eta+\left({ }_{0} D_{x}^{\beta} u\right) \eta_{u}-\left({ }_{0} D_{x}^{\beta} \xi+\left({ }_{0} D_{x}^{\beta} u\right) \xi_{u}\right)_{0} D_{x}^{\beta} u \\
& -\left({ }_{0} D_{x}^{\beta} \tau+\left({ }_{0} D_{x}^{\beta} u\right) \tau_{u}\right)_{0} D_{t}^{\alpha} u, \\
\eta^{t} & ={ }_{0} D_{t}^{\alpha} \eta+\left({ }_{0} D_{t}^{\alpha} u\right) \eta_{u}-\left({ }_{0} D_{t}^{\alpha} \xi+\left({ }_{0} D_{t}^{\alpha} u\right) \xi_{u}\right)_{0} D_{x}^{\beta} u \\
& -\left({ }_{0} D_{t}^{\alpha} \tau+\left({ }_{0} D_{t}^{\alpha} u\right) \tau_{u}\right){ }_{0} D_{t}^{\alpha} u, \\
\eta^{x x} & ={ }_{0} D_{x}^{2 \beta} \eta+\left({ }_{0} D_{x}^{\beta} u\right)\left({ }_{0} D_{x}^{\beta} \eta_{u}\right)-\left({ }_{0} D_{x}^{2 \beta} \xi\right. \\
& \left.+\left({ }_{0} D_{x}^{\beta} u\right)\left({ }_{0} D_{x}^{\beta} \xi_{u}\right)\right){ }_{0} D_{x}^{\beta} u-\left({ }_{0} D_{x}^{2 \beta} \tau\right. \\
& \left.+\left({ }_{0} D_{x}^{\beta} u\right)\left({ }_{0} D_{x}^{\beta} \tau_{u}\right)\right){ }_{0} D_{t}^{\alpha} u-2\left({ }_{0} D_{x}^{\beta} \tau\right. \\
& \left.+\left({ }_{0} D_{x}^{\beta} u\right) \tau_{u}\right){ }_{0} D_{t}^{\alpha}\left({ }_{0} D_{x}^{\beta} u\right)+\left({ }_{0} D_{x}^{2 \beta} u\right)\left(\eta_{u}\right. \\
& \left.-\left({ }_{0} D_{x}^{\beta} u\right) \xi_{u}-\left({ }_{0} D_{t}^{\alpha} u\right) \tau_{u}\right)-2\left({ }_{0} D_{x}^{\beta} \xi\right. \\
& \left.+\left({ }_{0} D_{x}^{\beta} u\right) \xi_{u}\right){ }_{0} D_{x}^{2 \beta} u+\left[{ }_{0} D_{x}^{\beta} \eta_{u}+\left({ }_{0} D_{x}^{\beta} u\right) \eta_{u u}\right. \\
& -\left({ }_{0} D_{x}^{\beta} \xi_{u}+\left({ }_{0} D_{x}^{\beta} u\right) \xi_{u u}\right){ }_{0} D_{x}^{\beta} u \\
& \left.-\left({ }_{0} D_{x}^{\beta} \tau_{u}+\left({ }_{0} D_{x}^{\beta} u\right) \tau_{u u}\right){ }_{0} D_{t}^{\alpha} u\right]{ }_{0} D_{x}^{\beta} u .
\end{aligned}
$$

Now, using the above generalized fractional prolongation vector fields in (12) and equating the coefficient of various derivative terms to zero, we get the following simplified set of determining equations:

$$
\begin{aligned}
& \tau_{u}=0, \\
& { }_{0} D_{x}^{\beta} \tau=0, \\
& \xi_{u}=0, \\
& 2 g(t){ }_{0} D_{x}^{\beta} \xi-g(t){ }_{0} D_{t}^{\alpha} \tau-\tau\left({ }_{0} D_{t}^{\alpha} g(t)\right)-g(t) \eta_{u} \\
& \quad-f(t) \eta_{u u}=0, \\
& 2 f(t){ }_{0} D_{x}^{\beta} \xi-f(t){ }_{0} D_{t}^{\alpha} \tau-\tau\left({ }_{0} D_{t}^{\alpha} f(t)\right)=0, \\
& f(t){ }_{0} D_{x}^{2 \beta} \xi-{ }_{0} D_{t}^{\alpha} \xi-2 g(t){ }_{0} D_{x}^{\beta} \eta \\
& \quad-2 f(t){ }_{0} D_{x}^{\beta} \eta_{u}=0, \\
& { }_{0} D_{t}^{\alpha} \eta-f(t){ }_{0} D_{x}^{2 \beta} \eta=0 .
\end{aligned}
$$


On solving (20) by using fractional Lie group method, we obtain a particular solution as

$$
\eta=a_{1} \frac{x^{\beta}}{\Gamma(1+\beta)}+a_{2}\left({ }_{0} D_{t}^{\alpha} F(t)\right)+a_{2} \frac{x^{2 \beta}}{\Gamma(1+2 \beta)}+a_{3},
$$

where ${ }_{0} D_{t}^{2 \alpha} F(t)={ }_{0} D_{t}^{\alpha} f(t)$ and $a_{1}, a_{2}$, and $a_{3}$ are arbitrary constants. Using this value of $\eta$ in (17) and (18), we get

$$
2(g(t)-f(t)){ }_{0} D_{x}^{2 \beta} \xi=0,
$$

which brings forth the following possibilities:

(i) ${ }_{0} D_{x}^{2 \beta} \xi=0$.

(ii) $g(t)=f(t)$.

Case (i). In this case, from the determining equations, we get

$$
\begin{aligned}
\xi= & -2 a_{1} G(t)-2 a_{2} G(t) \frac{x^{\beta}}{\Gamma(1+\beta)}+a_{4} \frac{x^{\beta}}{\Gamma(1+\beta)} \\
& +a_{5}, \\
\tau= & \frac{1}{{ }_{0} D_{t}^{2 \alpha} F(t)}-\left[4 a_{2} H(t)+2 a_{4}\left({ }_{0} D_{t}^{\alpha} F(t)\right)+a_{6}\right], \\
\eta= & a_{1} \frac{x^{\beta}}{\Gamma(1+\beta)}+a_{2}\left({ }_{0} D_{t}^{\alpha} F(t)\right)+a_{2} \frac{x^{2 \beta}}{\Gamma(1+2 \beta)}+a_{3},
\end{aligned}
$$

where ${ }_{0} D_{t}^{\alpha} G(t)=g(t),{ }_{0} D_{t}^{\alpha} H(t)=\left({ }_{0} D_{t}^{2 \alpha} F(t)\right) G(t)$, and $a_{1}$, $a_{2}, \ldots, a_{6}$ are six arbitrary constants. Using (23) in (17), we also get $g(t)=k f(t)$, where $k$ is an arbitrary constant. This covers case (ii); as for $f(t)=g(t)$ on solving the determining equations, we get ${ }_{0} D_{x}^{2 \beta} \xi=0$. Further, for $f(t)=g(t)=1$ and $\beta=1$, the infinitesimals can be reduced to those reported in [32] by setting the coefficients $a_{1}=-c_{5}, a_{2}=-2 c_{6}, a_{3}=c_{3}$, $a_{4}=c_{4}, a_{5}=c_{1}$, and $a_{6}=c_{2}$.

Hence, the fractional point symmetry generators admitted by (1) are given by

$$
\begin{aligned}
V_{1}= & -2 G(t) \frac{\partial^{\beta}}{\partial x^{\beta}}+\frac{x^{\beta}}{\Gamma(1+\beta)} \frac{\partial}{\partial u}, \\
V_{2}= & -2 G(t) \frac{x^{\beta}}{\Gamma(1+\beta)} \frac{\partial^{\beta}}{\partial x^{\beta}}-\frac{4 H(t)}{{ }_{0} D_{t}^{2 \alpha} F(t)} \frac{\partial^{\alpha}}{\partial t^{\alpha}} \\
& +\left[{ }_{0} D_{t}^{\alpha} F(t)+\frac{x^{2 \beta}}{\Gamma(1+2 \beta)} \frac{\partial}{\partial u}\right], \\
V_{3}= & \frac{\partial}{\partial u}, \\
V_{4}= & \frac{x^{\beta}}{\Gamma(1+\beta)} \frac{\partial^{\beta}}{\partial x^{\beta}}+\frac{2\left({ }_{0} D_{t}^{\alpha} F(t)\right)}{{ }_{0} D_{t}^{2 \alpha} F(t)} \frac{\partial^{\alpha}}{\partial t^{\alpha}}, \\
V_{5}= & \frac{\partial^{\beta}}{\partial x^{\beta}}, \\
V_{6}= & \frac{1}{{ }_{0} D_{t}^{2 \alpha} F(t)} \frac{\partial^{\alpha}}{\partial t^{\alpha}} .
\end{aligned}
$$

TABLE 1

\begin{tabular}{ccccccc}
\hline & $V_{1}$ & $V_{2}$ & $V_{3}$ & $V_{4}$ & $V_{5}$ & $V_{6}$ \\
\hline$V_{1}$ & 0 & 0 & 0 & $V_{1}$ & $-V_{3}$ & $2 V_{5}$ \\
$V_{2}$ & 0 & 0 & 0 & $2 V_{2}$ & $2 V_{1}$ & $4 V_{4}-2 V_{3}$ \\
$V_{3}$ & 0 & 0 & 0 & 0 & 0 & 0 \\
$V_{4}$ & $-V_{1}$ & $-2 V_{2}$ & 0 & 0 & $-V_{5}$ & $2 V_{6}$ \\
$V_{5}$ & $V_{3}$ & $-2 V_{1}$ & 0 & $V_{5}$ & 0 & 0 \\
$V_{6}$ & $-2 V_{5}$ & $2 V_{3}-4 V_{4}$ & 0 & $-2 V_{6}$ & 0 & 0 \\
\hline
\end{tabular}

These infinitesimal generators can be used to determine a six-parameter fractional Lie group of point transformation acting on $(x, t, u)$-space. It can be verified easily that the set $\left\{V_{1}, V_{2}, V_{3}, V_{4}, V_{5}, V_{6}\right\}$ forms a six-dimensional Lie algebra under the Lie bracket $[X, Y]=X Y-Y X$, which reduces to the well-known generalized Galilea algebra [26] for $\alpha=\beta=1$. The commutator table is as given in Table 1.

Further, from the commutator table, it can be seen that the sets $\left\{V_{3}\right\}$ and $\left\{V_{1}, V_{2}, V_{3}\right\}$ form solvable subalgebra. Also, $V_{3}$ is the centre of the six-dimensional Lie algebra as it commutes with every element of the Lie algebra. The group transformation generated by the infinitesimal generators $V_{i}, i=1,2, \ldots, 6$, is obtained by solving the system of ordinary differential equations:

$$
\begin{aligned}
\frac{(d \tilde{x})^{\beta}}{\Gamma(1+\beta) d \epsilon} & =\xi(\tilde{x}, \tilde{t}, \tilde{u}), \\
\frac{(d \tilde{t})^{\alpha}}{\Gamma(1+\alpha) d \epsilon} & =\tau(\tilde{x}, \tilde{t}, \tilde{u}), \\
\frac{d \tilde{u}}{d \epsilon} & =\eta(\tilde{x}, \tilde{t}, \tilde{u}),
\end{aligned}
$$

with the initial conditions

$$
\begin{aligned}
& \left.\tilde{x}\right|_{\epsilon=0}=x, \\
& \left.\widetilde{t}\right|_{\epsilon=0}=t, \\
& \left.\widetilde{u}\right|_{\epsilon=0}=u .
\end{aligned}
$$

Exponentiating the infinitesimal symmetries of (1), we get the one-parameter groups $g_{i}(\epsilon)$ generated by $V_{i}, i=1,2, \ldots, 6$ :

$$
\begin{aligned}
g_{1} & :\left(\frac{x^{\beta}}{\Gamma(1+\beta)}, \frac{t^{\alpha}}{\Gamma(1+\alpha)}, u\right) \rightarrow\left(\frac{x^{\beta}}{\Gamma(1+\beta)}\right. \\
& \left.-\frac{2 \epsilon G}{\Gamma(1+\alpha)}, \frac{t^{\alpha}}{\Gamma(1+\alpha)}, u+\frac{\epsilon x^{\beta}}{\Gamma(1+\beta)}\right), \\
g_{2}: & \left(\frac{x^{\beta}}{\Gamma(1+\beta)}, \frac{t^{\alpha}}{\Gamma(1+\alpha)}, u\right) \\
& \longrightarrow\left(\frac{x^{\beta}}{\Gamma(1+\beta)} e^{-2 \epsilon G}, \frac{G}{(1+2 \epsilon G)}, u+\frac{\epsilon\left({ }_{0} D_{t}^{\alpha} F(t)\right)}{(1+2 \epsilon G)}\right. \\
& \left.+\frac{\epsilon x^{2 \beta}}{\Gamma(1+2 \beta)} e^{-4 \epsilon G /(1+2 \epsilon G)}\right),
\end{aligned}
$$




$$
\begin{aligned}
& g_{3}:\left(\frac{x^{\beta}}{\Gamma(1+\beta)}, \frac{t^{\alpha}}{\Gamma(1+\alpha)}, u\right) \\
& \longrightarrow\left(\frac{x^{\beta}}{\Gamma(1+\beta)}, \frac{t^{\alpha}}{\Gamma(1+\alpha)}, u+\epsilon\right), \\
& g_{4}:\left(\frac{x^{\beta}}{\Gamma(1+\beta)}, \frac{t^{\alpha}}{\Gamma(1+\alpha)}, u\right) \\
& \quad \longrightarrow\left(\frac{e^{\epsilon} x^{\beta}}{\Gamma(1+\beta)}, \frac{e^{2 \epsilon} t^{\alpha}}{\Gamma(1+\alpha)}, u\right), \\
& g_{5}:\left(\frac{x^{\beta}}{\Gamma(1+\beta)}, \frac{t^{\alpha}}{\Gamma(1+\alpha)}, u\right) \longrightarrow\left(\frac{x^{\beta}}{\Gamma(1+\beta)}\right. \\
&\left.+\epsilon, \frac{t^{\alpha}}{\Gamma(1+\alpha)}, u\right), \\
& g_{6}:\left(\frac{x^{\beta}}{\Gamma(1+\beta)}, \frac{t^{\alpha}}{\Gamma(1+\alpha)}, u\right) \\
& \quad \longrightarrow\left(\frac{x^{\beta}}{\Gamma(1+\beta)}, \frac{t^{\alpha}}{\Gamma(1+\alpha)}+\epsilon, u\right) .
\end{aligned}
$$

Now, since $g_{i}$ is a symmetry, if $u=\chi\left(x^{\beta} / \Gamma(1+\beta), t^{\alpha} / \Gamma(1+\alpha)\right)$ is a solution of (1), following $u_{i}$ are also solutions of (1):

$$
\begin{aligned}
u_{1}= & \chi\left(\frac{x^{\beta}}{\Gamma(1+\beta)}-\frac{2 \epsilon G}{\Gamma(1+\alpha)}, \frac{t^{\alpha}}{\Gamma(1+\alpha)}\right) \\
& -\frac{\epsilon x^{\beta}}{\Gamma(1+\beta)}, \\
u_{2}= & \chi\left(\frac{x^{\beta}}{\Gamma(1+\beta)} e^{-2 \epsilon G}, \frac{G}{(1+2 \epsilon G)}\right)-\frac{\epsilon\left({ }_{0} D_{t}^{\alpha} F(t)\right)}{(1+2 \epsilon G)} \\
& -\frac{\epsilon x^{2 \beta}}{\Gamma(1+2 \beta)} e^{-4 \epsilon G /(1+2 \epsilon G)}, \\
u_{3}= & \chi\left(\frac{x^{\beta}}{\Gamma(1+\beta)}, \frac{t^{\alpha}}{\Gamma(1+\alpha)}\right)-\epsilon, \\
u_{4}= & \chi\left(\frac{e^{\epsilon} x^{\beta}}{\Gamma(1+\beta)}, \frac{e^{2 \epsilon} t^{\alpha}}{\Gamma(1+\alpha)}\right), \\
u_{5}= & \chi\left(\frac{x^{\beta}}{\Gamma(1+\beta)}+\epsilon, \frac{t^{\alpha}}{\Gamma(1+\alpha)}\right), \\
u_{6}= & \chi\left(\frac{x^{\beta}}{\Gamma(1+\beta)}, \frac{t^{\alpha}}{\Gamma(1+\alpha)}+\epsilon\right) .
\end{aligned}
$$

\section{Some Exact Solutions of the Space-Time Fractional Burgers' Equation}

In this section, we investigate some exact solutions of (1) corresponding to following infinitesimal generators: (i) $V_{1}$

(ii) $V_{4}$

(iii) $n V_{5}+m V_{3}$

(iv) $r V_{5}+V_{6}$ and

(v) $s V_{3}+V_{6}$

$r, s, m$, and $n$ are nonzero arbitrary constant parameters.

Theorem 1. Under the group of transformations $T(x, t)=t^{\alpha} /$ $\Gamma(1+\alpha)$ and $\phi(T)=x^{2 \beta} / \Gamma(1+2 \beta)+2 G(t) u(x, t)$, Burgers' equation (1) reduces to a linear differential equation of first order: $\phi^{\prime}(T)-H_{1}(T) \phi(T)=-H_{2}(T)$, where $H_{1}(T)={ }_{0} D_{t}^{\alpha} G(t) / G(t)$ and $H_{2}(T)={ }_{0} D_{t}^{2 \alpha} F(t)$, which admits a solution given by $u(x, t)=(1 / 2 G(t))\left[e^{\int H_{1} d T}\left(k_{1}-\int H_{2} e^{-\int H_{1} d T} d T\right)-x^{2 \beta} / \Gamma(1+\right.$ $2 \beta)$, where $k_{1}$ is an arbitrary constant.

Proof. Consider the infinitesimal generator $V_{1}$, given by

$$
V_{1}=-2 G(t) \frac{\partial^{\beta}}{\partial x^{\beta}}+\frac{x^{\beta}}{\Gamma(1+\beta)} \frac{\partial}{\partial u} .
$$

We find the resulting invariant solution by reducing (1) to a linear ordinary differential equation (32) using differential invariants. The fractional characteristic equations for $V_{1}$ are

$$
\frac{(d x)^{\beta} / \Gamma(1+\beta)}{-2 G(t)}=\frac{(d t)^{\alpha} / \Gamma(1+\alpha)}{0}=\frac{d u}{x^{\beta} / \Gamma(1+\beta)} .
$$

On solving the above fractional characteristic equations, we obtain two functionally independent invariants as $T=$ $t^{\alpha} / \Gamma(1+\alpha)$ and $\nu=x^{2 \beta} / \Gamma(1+2 \beta)+2 G(t) u$.

Now the solution of the fractional characteristic equations will be of the form $v=\phi(T)$; therefore

$$
u=\frac{1}{2 G(T)}\left[\phi(T)-\frac{x^{2 \beta}}{\Gamma(1+2 \beta)}\right] .
$$

Substituting this value of $u$ in (1), we get the reduced linear ordinary differential equation as

$$
\phi^{\prime}(T)-H_{1}(T) \phi(T)=-H_{2}(T),
$$

where $T=t^{\alpha} / \Gamma(1+\alpha), H_{1}(T)={ }_{0} D_{t}^{\alpha} G(t) / G(t)$, and $H_{2}(T)=$ ${ }_{0} D_{t}^{2 \alpha} F(t)$. On solving (32), we obtain $\phi(T)=e^{\int H_{1} d T}\left(k_{1}-\right.$ $\left.\int H_{2} e^{-\int H_{1} d T} d T\right)$, where $k_{1}$ is an arbitrary constant. This gives

$$
\begin{aligned}
& u(x, t)=\frac{1}{2 G(t)}\left[e^{\int H_{1} d T}\left(k_{1}-\int H_{2} e^{-\int H_{1} d T} d T\right)\right. \\
& \left.-\frac{x^{2 \beta}}{\Gamma(1+2 \beta)}\right] .
\end{aligned}
$$

Theorem 2. The similarity transformations $u(x, t)=\psi(X)$ along with the similarity variable $X(x, t)=x^{2 \beta} /(\Gamma(1+\beta))^{2}$ $\left({ }_{0} D_{t}^{\alpha} F(t)\right)$ reduce fractional Burgers' equation (1) to a nonlinear 
ordinary differential equation $\psi^{\prime \prime}(X)+(g(t) / f(t))\left(\psi^{\prime}(X)\right)^{2}+$ $(1 / 4) \psi^{\prime}(X)+(1 / 2 X) \psi^{\prime}(X)=0$, which leads to the solution $u(x, t)=(1 / k) \log \left\{k_{2}+2 \sqrt{\pi} k \operatorname{erf}\left(x^{\beta} / 2 \Gamma(1+\beta) \sqrt{\left({ }_{0} D_{t}^{\alpha} F(t)\right)}\right)\right\}$ $+k_{3}$, where $k_{2}$ and $k_{3}$ are arbitrary constants.

Proof. Let us consider the infinitesimal generator

$$
V_{4}=\frac{x^{\beta}}{\Gamma(1+\beta)} \frac{\partial^{\beta}}{\partial x^{\beta}}+\frac{2\left({ }_{0} D_{t}^{\alpha} F(t)\right)}{{ }_{0} D_{t}^{2 \alpha} F(t)} \frac{\partial^{\alpha}}{\partial t^{\alpha}} .
$$

Here the fractional characteristic equations give the invariants $X(x, t)=x^{2 \beta} /(\Gamma(1+\beta))^{2}\left({ }_{0} D_{t}^{\alpha} F(t)\right)$ and $u(x, t)=\psi(X)$. On substituting the above invariants in (1), it becomes a nonlinear ordinary differential equation of second order:

$$
\begin{aligned}
& \psi^{\prime \prime}(X)+\frac{g(t)}{f(t)}\left(\psi^{\prime}(X)\right)^{2}+\frac{1}{4} \psi^{\prime}(X)+\frac{1}{2 X} \psi^{\prime}(X) \\
& \quad=0
\end{aligned}
$$

which leads to the solution

$$
\begin{aligned}
& u(x, t)=\frac{1}{k} \log \left\{k_{2}\right. \\
& \left.+2 \sqrt{\pi} k \operatorname{erf}\left(\frac{x^{\beta}}{2 \Gamma(1+\beta) \sqrt{\left({ }_{0} D_{t}^{\alpha} F(t)\right)}}\right)\right\}+k_{3} .
\end{aligned}
$$

Theorem 3. Under the transformations $\zeta(x, t)=t^{\alpha} / \Gamma(1+\alpha)$ and $\varphi(\zeta)=x^{\beta} / \Gamma(1+\beta)-(n / m) u(x, t)$, fractional Burgers' equation (1) reduces to an ordinary fractional differential equation ${ }_{0} D_{t}^{\alpha} \varphi(t)+(m / n)\left({ }_{0} D_{t}^{\alpha} G(t)\right)=0$, which has the general solution as $u(x, t)=(m / n)\left[x^{\beta} / \Gamma(1+\beta)+(m / n) G(t)-\right.$ $\left.k_{4}\right]$, with $k_{4}$ being an arbitrary constant.

Proof. In this case, we study the infinitesimal generator

$$
V=n V_{5}+m V_{3}=n \frac{\partial^{\beta}}{\partial x^{\beta}}+m \frac{\partial}{\partial u} .
$$

The following invariants can be derived easily: $\zeta(x, t)=t^{\alpha} /$ $\Gamma(1+\alpha)$ and $\nu=x^{\beta} / \Gamma(1+\beta)-(n / m) u(x, t)$ and the reduced form of (1) as

$$
{ }_{0} D_{t}^{\alpha} \varphi(t)+\frac{m}{n}\left({ }_{0} D_{t}^{\alpha} G(t)\right)=0 .
$$

This easily yields the solution

$$
u(x, t)=\frac{m}{n}\left[\frac{x^{\beta}}{\Gamma(1+\beta)}+\frac{m}{n} G(t)-k_{4}\right] .
$$

Theorem 4. Under the transformations $\mu(x, t)=(1 / r)$ $\left(x^{\beta} / \Gamma(1+\beta)\right)-{ }_{0} D_{t}^{\alpha} F(t) / \Gamma(1+\alpha)$ and $\omega(\mu)=u(x, t)$, fractional Burgers' equation (1) reduces to a nonlinear ordinary differential equation $\omega^{\prime \prime}(\mu)+k \omega^{\prime 2}(\mu)+\left(r^{2} / \Gamma(1+\right.$ $\alpha) \omega^{\prime}(\mu)=0$, which admits the solution $u(x, t)=$

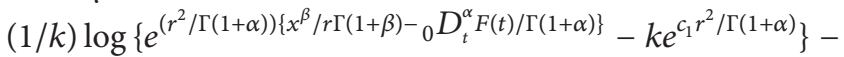
$\left(r^{2} / \Gamma(1+\alpha)\right)\left\{x^{\beta} / r \Gamma(1+\beta)-{ }_{0} D_{t}^{\alpha} F(t) / \Gamma(1+\alpha)\right\}+c_{2}$, where $c_{1}$ and $c_{2}$ are arbitrary constants.

Proof. Results can be easily derived by solving the fractional characteristic equations for the infinitesimal generator $r V_{5}+$ $V_{6}$.

Theorem 5. The similarity transformations $\gamma(x, t)=x^{\beta} / \Gamma(1+$ $\beta)$ and $\rho(\gamma)={ }_{0} D_{t}^{\alpha} F(t)-(\Gamma(1+\alpha) / s) u$ reduce Burgers' equation (1) to a nonlinear ordinary differential equation $\rho^{\prime \prime}(\gamma)-$ $(k s / \Gamma(1+\alpha))\left(\rho^{\prime}(\gamma)\right)^{2}+1=0$, which has the general solution as $u(x, t)=(s / \Gamma(1+\alpha))\left[{ }_{0} D_{t}^{\alpha} F(\mathrm{t})+(1 / k) \log \left\{\cosh \sqrt{k}\left(c_{3}+\right.\right.\right.$ $\left.\left.\left.x^{\beta} / \Gamma(1+\beta)\right)\right\}-c_{4}\right]$, where $c_{3}$ and $c_{4}$ are arbitrary constants.

Proof. The proof is very much similar to the previous theorems.

\section{Some Exact Solutions of Fractional Potential Burgers' Equation by the Invariant Subspace Method}

The invariant subspace method was introduced by Galaktionov [38] in order to discover exact solutions of nonlinear partial differential equations. The method was further applied by Gazizov and Kasatkin [39] and Sahadevan [40] to some nonlinear fractional-order differential equations. Here, we give a brief description of the method.

Consider the fractional evolution equation ${ }_{0} D_{t}^{\alpha} u=F[u]$, where $u=u(x, t)$ and $F[u]$ is a nonlinear differential operator.

The $n$-dimensional linear space $W_{n}=\left\langle f_{1}(x), \ldots, f_{n}(x)\right\rangle$ is called invariant under the operator $F[u]$, if $F[u] \epsilon$ $W_{n}$ for any $u \in W_{n}$, which means there exist $n$ functions $\phi_{1}, \ldots, \phi_{n}$ such that $F\left[C_{1} f_{1}(x)+\cdots+C_{n} f_{n}(x)\right]=$ $\phi_{1}\left(C_{1}, \ldots, C_{n}\right) f_{1}(x)+\cdots+\phi_{n}\left(C_{1}, \ldots, C_{n}\right) f_{n}(x)$, where $C_{1}$, $\ldots, C_{n}$ are arbitrary constants and $\left\{\phi_{n}\right\}$ are the expansion coefficients of $F[u] \in W_{n}$ in the basis $\left\{f_{i}\right\}$. It follows that an exact solution of fractional evolution equation can be obtained as $u(x, t)=\sum_{i=1}^{n} a_{i}(t) f_{i}(x)$, where the coefficient functions $a_{1}(t), a_{2}(t), \ldots, a_{n}(t)$ satisfy a system of fractional ODEs:

$$
\begin{aligned}
&{ }_{0} D_{t}^{\alpha} a_{i}(t)=\phi_{i}\left(a_{1}(t), a_{2}(t), \ldots, a_{n}(t)\right) \\
& \\
& i=1,2, \ldots, n
\end{aligned}
$$

For further details, the reader is referred to [40].

$$
\text { For (1), } F[u]={ }_{0} D_{t}^{\alpha} u-f(t){ }_{0} D_{x}^{2 \beta} u-g(t)\left({ }_{0} D_{x}^{\beta} u\right)^{2} \text {. We }
$$
have the space $W_{3}=\left\langle 1, x^{\beta} / \Gamma(1+\beta), x^{2 \beta} / \Gamma(1+2 \beta)\right\rangle$ as invariant under $F[u]$, if and only if, for any $u(x, t)=a_{1}(t)+$ 
$a_{2}(t)\left(x^{\beta} / \Gamma(1+\beta)\right)+a_{3}(t)\left(x^{2 \beta} / \Gamma(1+2 \beta)\right) \in W_{3}, F[u] \in W_{3}$ or equivalently if

$$
\begin{array}{r}
a_{3}(t) f(t)+\left(a_{2}(t)\right)^{2} g(t)=b_{1}, \\
\left(a_{3}(t)\right)^{2} g(t)=b_{2}, \\
a_{2}(t) a_{3}(t) g(t)=b_{3},
\end{array}
$$

where $b_{1}, b_{2}$, and $b_{3}$ are arbitrary constants. This allows us to consider an exact solution of (1) as

$$
u(x, t)=a_{1}(t)+a_{2}(t) \frac{x^{\beta}}{\Gamma(1+\beta)}+a_{3}(t) \frac{x^{2 \beta}}{\Gamma(1+2 \beta)} .
$$

Substituting the value of $u(x, t)$ from (42) into (1) and equating the coefficients of the elements of space $W_{3}=$ $\left\langle 1, x^{\beta} / \Gamma(1+\beta), x^{2 \beta} / \Gamma(1+2 \beta)\right\rangle$, we get the following system of fractional differential equations:

$$
\begin{aligned}
& { }_{0} D_{t}^{\alpha} a_{3}(t)=\frac{\Gamma(1+2 \beta)}{(\Gamma(1+\beta))^{2}} g\left(a_{3}(t)\right)^{2}, \\
& { }_{0} D_{t}^{\alpha} a_{2}(t)=2 a_{2}(t) a_{3}(t) g, \\
& { }_{0} D_{t}^{\alpha} a_{1}(t)=f a_{3}(t)+g\left(a_{2}(t)\right)^{2} .
\end{aligned}
$$

Equations (43) can be readily solved to yield

$$
\begin{aligned}
-\frac{1}{a_{3}(t)} & =\frac{\Gamma(1+2 \beta)}{(\Gamma(1+\beta))^{2}}\left({ }_{0} I_{t}^{\alpha} g\right), \\
\log a_{2}(t) & =2\left({ }_{0} I_{t}^{\alpha}\left(a_{3}(t) g\right)\right), \\
a_{1}(t) & ={ }_{0} I_{t}^{\alpha}\left(a_{3}(t) f+\left(a_{2}(t)\right)^{2} g\right),
\end{aligned}
$$

where the functions $f(t)$ and $g(t)$ are integrable in the sense of Riemann-Liouville and ${ }_{0} I_{t}^{\alpha} g(t) \neq 0$.

On solving (44), we get

$$
\begin{aligned}
& a_{3}(t)=\frac{B}{{ }_{0} I_{t}^{\alpha} g(t)}, \\
& a_{2}(t)=\left({ }_{0} I_{t}^{\alpha} g(t)\right)^{2 B}, \\
& a_{1}(t)={ }_{0} I_{t}^{\alpha}\left[g(t)\left({ }_{0} I_{t}^{\alpha} g(t)\right)^{4 B}+\frac{B f(t)}{{ }_{0} I_{t}^{\alpha} g(t)}\right],
\end{aligned}
$$

where $B=-(\Gamma(1+\beta))^{2} / \Gamma(1+2 \beta)$.

Using (42) and (45), one can easily obtain an exact solution of (1).

\section{Conclusion}

The main purpose of Lie symmetry method is to reduce PDEs to ODEs by introducing suitable similarity variable and similarity solutions. Here, in this paper, the authors show that the fractional potential Burgers' equation possesses similarity solutions exactly as its counterparts with integerorder derivatives. By using conveniently defined similarity variables, fractional potential Burgers' equation reduces to ordinary differential equations which are further solved to derive some group invariant solutions. The authors also utilised the invariant subspace method to deduce some exact solutions of fractional potential Burgers' equation. Software like Mathematica and Maple has been utilised in solving some ordinary differential equations.

\section{Competing Interests}

The authors declare that they have no competing interests.

\section{Acknowledgments}

One of the authors (Manoj Gaur) would like to thank the University Grants Commission (UGC), New Delhi, India, for providing Research Fellowship under the scheme UGC-CSIR NET JRF in Science, Humanities \& Social Sciences.

\section{References}

[1] H. Brezis and F. Browder, "Partial differential equations in the 20th century," Advances in Mathematics, vol. 135, no. 1, pp. 76144,1998

[2] L. Debtnath, Nonlinear Partial Differential Equations for Scientist and Engineers, Birkhäuser, Boston, Mass, USA, 1997.

[3] J. D. Cole, "On a quasi-linear parabolic equation occurring in aerodynamics," Quarterly of Applied Mathematics, vol. 9, pp. 225-236, 1951.

[4] E. Barkai, "Fractional Fokker-Planck equation, solution, and application," Physical Review E, vol. 63, no. 4, Article ID 46118, 2001.

[5] J. H. He, "Some applications of nonlinear fractional differential equations and their approximations," Bulletin of Science, Technology \& Society, vol. 15, no. 2, pp. 86-90, 1999.

[6] H. M. Jaradat, F. Awawdeh, S. Al-Shara, M. Alquran, and S. Momani, "Controllable dynamical behaviors and the analysis of fractal Burgers hierarchy with the full effects of inhomogeneities of media," Romanian Journal of Physics, vol. 60, no. 3-4, pp. 324343, 2015.

[7] K. S. Miller and B. Ross, An Introduction to the Fractional Calculus and Fractional Differential Equations, John Wiley \& Sons, New York, NY, USA, 1993.

[8] K. B. Oldham and J. Spanier, The Fractional Calculus, Academic Press, New York, NY, USA, 1974.

[9] B. West, M. Bologna, and P. Grigolini, Physics of Fractal Operators, Springer Science \& Business Media, New York, NY, USA, 2012.

[10] R. Caponetto, G. Dongola, L. Fortuna, and I. Petr, Fractional Order Systems (Modelling and Control Applications), World Scientific, Singapore, 2010.

[11] I. Podlubny, Fractional Differential Equations: An Introduction to Fractional Derivatives, Fractional Differential Equations, to Methods of Their Solution and Some of Their Applications, vol. 198, Academic press, Cambridge, Mass, USA, 1998.

[12] K. Al-Khaled and M. Alquran, "An approximate solution for a fractional model of generalized Harry Dym equation," Mathematical Sciences, vol. 8, no. 4, pp. 125-130, 2014.

[13] M. Alquran, K. Al-Khaled, T. Sardar, and J. Chattopadhyay, "Revisited Fisher's equation in a new outlook: a fractional 
derivative approach," Physica A. Statistical Mechanics and Its Applications, vol. 438, pp. 81-93, 2015.

[14] M. Alquran, "Analytical solution of time-fractional twocomponent evolutionary system of order 2 by residual power series method," The Journal of Applied Analysis and Computation, vol. 5, no. 4, pp. 589-599, 2015.

[15] A. Arikoglu and I. Ozkol, "Solution of fractional differential equations by using differential transform method," Chaos, Solitons \& Fractals, vol. 34, no. 5, pp. 1473-1481, 2007.

[16] Y. Chen and H.-L. An, "Numerical solutions of coupled Burgers equations with time- and space-fractional derivatives," Applied Mathematics and Computation, vol. 200, no. 1, pp. 87-95, 2008.

[17] R. K. Gazizov, A. A. Kasatkin, and S. Yu Lukashchuk, "Continuous transformation groups of fractional differential equations," Vestnik USATU, vol. 9, no. 3 (21), pp. 125-135, 2007.

[18] M. Klimek and O. P. Agrawal, "Space- and time-fractional legendre-pearson diffusion equation," in Proceedings of the ASME International Design Engineering Technical Conferences and Computers and Information in Engineering Conference (IDETC/CIE '13), August 2013.

[19] Z.-B. Li and J.-H. He, "Fractional complex transform for fractional differential equations," Mathematical \& Computational Applications, vol. 15, no. 5, pp. 970-973, 2010.

[20] M. Merdan and A. Gökdoğan, "Solution of nonlinear oscillators with fractional nonlinearities by using the modified differential transformation method," Mathematical and Computational Applications, vol. 16, no. 3, pp. 761-772, 2011.

[21] S. Momani and K. Al-Khaled, "Numerical solutions for systems of fractional differential equations by the decomposition method," Applied Mathematics and Computation, vol. 162, no. 3 , pp. 1351-1365, 2005.

[22] Z. Odibat and S. Momani, "Numerical solution of FokkerPlanck equation with space- and time-fractional derivatives," Physics Letters, Section A: General, Atomic and Solid State Physics, vol. 369, no. 5-6, pp. 349-358, 2007.

[23] B. Ross, Fractional Calculus and Its Applications: Proceedings of the International Conference Held at the University of New Haven, June 1974, Springer, Berlin, Germany, 1975.

[24] S. G. Samko, A. A. Kilbas, and O. I. Marichev, Fractional Integrals and Derivatives, Gordon and Breach, London, UK, 1993.

[25] N. H. Ibragimov, CRC Handbook of Lie Group Analysis of Differential Equations, vol. 3, CRC Press, 1995.

[26] P. J. Olver, Applications of Lie Groups to Differential Equations, vol. 107 of Graduate Texts in Mathematics, Springer Science \& Business Media, New York, NY, USA, 2000.

[27] T. M. Atanacković, S. Konjik, S. Pilipović, and S. Simić, "Variational problems with fractional derivatives: invariance conditions and Nöther's theorem," Nonlinear Analysis: Theory, Methods \& Applications, vol. 71, no. 5-6, pp. 1504-1517, 2009.

[28] V. D. Djordjevic and T. M. Atanackovic, "Similarity solutions to nonlinear heat conduction and Burgers/Korteweg-de Vries fractional equations," Journal of Computational and Applied Mathematics, vol. 222, no. 2, pp. 701-714, 2008.

[29] M. Gaur and K. Singh, "On group invariant solutions of fractional order Burgers-Poisson equation," Applied Mathematics and Computation, vol. 244, pp. 870-877, 2014.

[30] H. Liu, "Complete group classifications and symmetry reductions of the fractional fifth-order KdV types of equations," Studies in Applied Mathematics, vol. 131, no. 4, pp. 317-330, 2013.
[31] M. S. Hashemi, "Group analysis and exact solutions of the time fractional Fokker-Planck equation," Physica A: Statistical Mechanics and its Applications, vol. 417, pp. 141-149, 2015.

[32] G.-C. Wu, "A fractional Lie group method for anomalous diffusion equations," Communications in Fractional Calculus, vol. 1, pp. 27-31, 2010.

[33] G. Jumarie, "Modified Riemann-Liouville derivative and fractional Taylor series of nondifferentiable functions further results," Computers and Mathematics with Applications, vol. 51, no. 9-10, pp. 1367-1376, 2006.

[34] J. Guy, "Lagrange characteristic method for solving a class of nonlinear partial differential equations of fractional order," Applied Mathematics Letters, vol. 19, no. 9, pp. 873-880, 2006.

[35] G.-C. Wu, "Lie group classifications and non-differentiable solutions for time-fractional Burgers equation," Communications in Theoretical Physics, vol. 55, no. 6, pp. 1073-1076, 2011.

[36] G. Jumarie, "From self-similarity to fractional derivative of nondifferentiable functions via Mittag-Leffler function," Applied Mathematical Sciences, vol. 2, no. 37-40, pp. 1949-1962, 2008.

[37] G. Jumarie, Fractional Differential Calculus for Non-Differentiable Functions: Mechanics, Geometry, Stochastics, Information Theory, Lambert Academic Publishing, Saarbrucken, Germany, 2013.

[38] V. A. Galaktionov, S. A. Posashkov, and S. R. Svirshchevskii, Exact Solutions and Invariant Subspaces of Nonlinear Partial Differential Equations in Mechanics and Physics, CRC Press, Boca Raton, Fla, USA, 2006.

[39] R. K. Gazizov and A. A. Kasatkin, "Construction of exact solutions for fractional order differential equations by the invariant subspace method," Computers \& Mathematics with Applications, vol. 66, no. 5, pp. 576-584, 2013.

[40] R. Sahadevan and T. Bakkyaraj, "Invariant subspace method and exact solutions of certain nonlinear time fractional partial differential equations," Fractional Calculus and Applied Analysis, vol. 18, no. 1, pp. 146-162, 2015. 


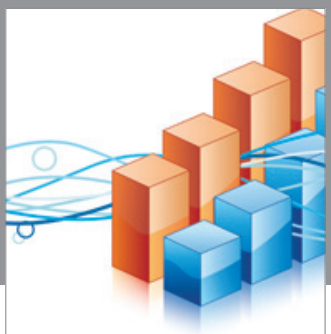

Advances in

Operations Research

vatem alat4

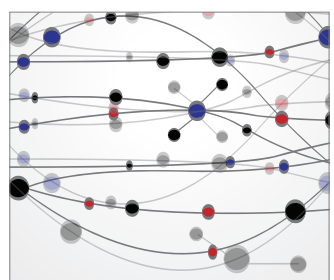

\section{The Scientific} World Journal
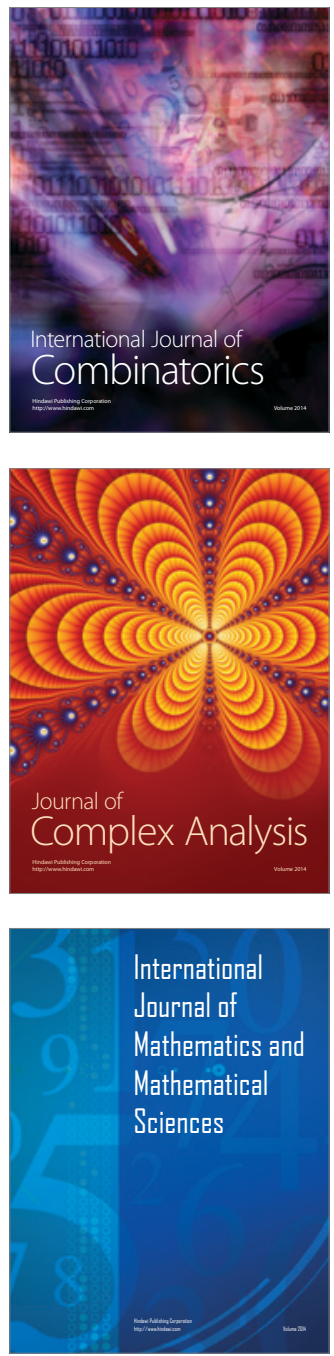
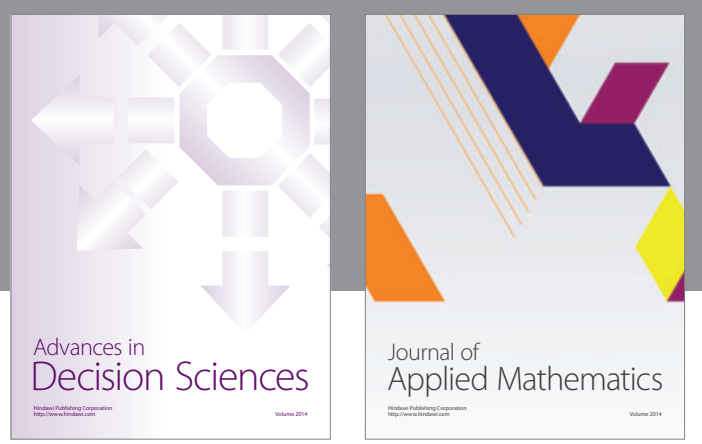

Algebra

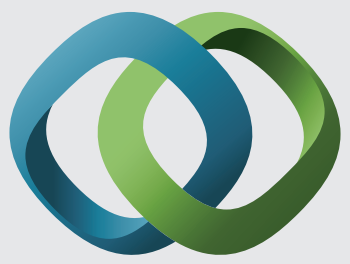

\section{Hindawi}

Submit your manuscripts at

http://www.hindawi.com
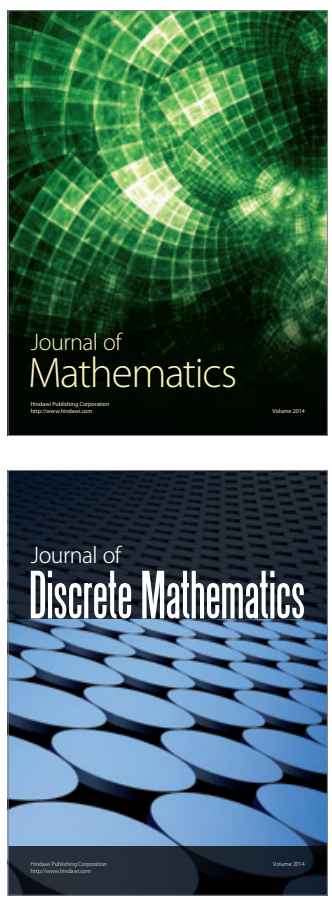

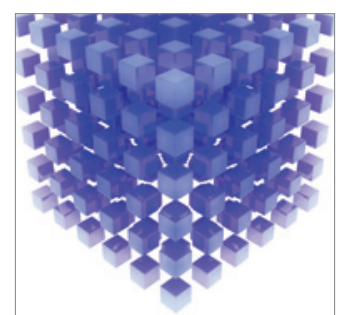

Mathematical Problems in Engineering
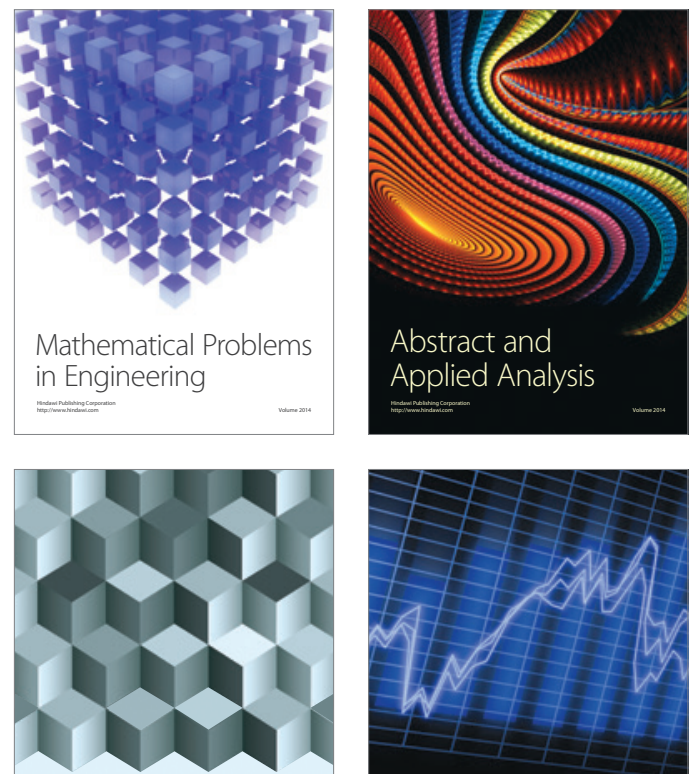

Journal of

Function Spaces

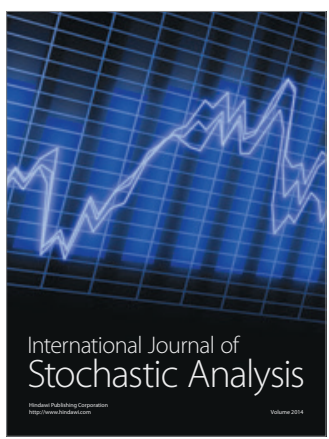

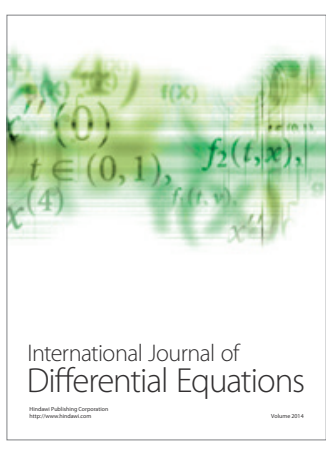
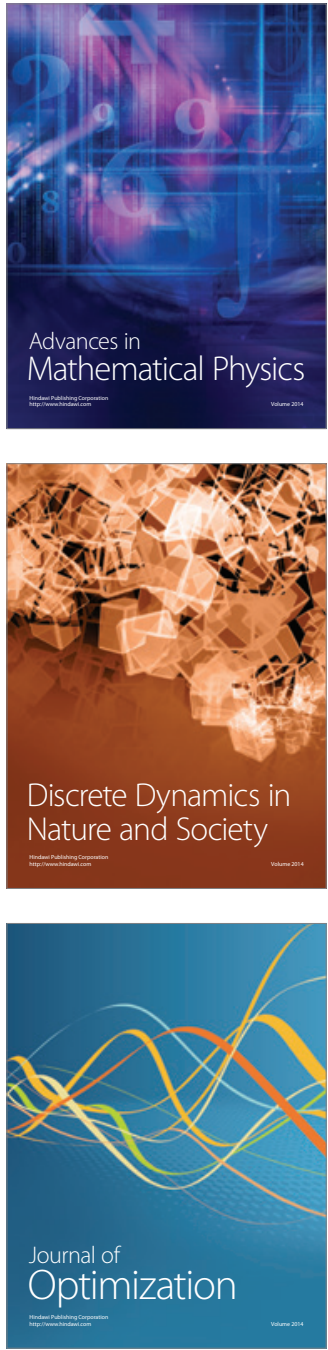